\title{
Deep learning approach to analyse, detect and classify COVID-19 patients
}

\author{
Hakan Koyuncu ${ }^{1}$ and Dinesh Sharma ${ }^{2}$ \\ ${ }^{1}$ Altinbas University, Computer Engineering Dept.,34217,Istanbul, Turkey \\ ${ }^{2}$ Amity University, Computer Engineering Dept., 201301, Gwalior, India
}

Received: 13 June 2021, Accepted: 16 June 2021

Published online: 4 July 2021.

\begin{abstract}
An early-stage classification model is presented to classify between the COVID and non-COVID patients. World health organization (WHO) and many governmental and private organizations have presented a significant approach in solving the world pandemic problem. In this study, the analysis, detection, and classification of COVID-19 patients with the help of the different worldwide chest $\mathrm{x}$-ray dataset and for the prediction, different updated statistics of COVID-19 cases have been used all over the world. The dataset consists of different COVID and non-COVID patients? x-ray details which will be further used for classification purposes. Different machine learning approaches were deployed like image filtration, image enhancement, feature extraction, detection, and evaluation. A deep learning classification approach was introduced by using the AdaBoost integrated with Convolution neural network (CNN) for the detection and evaluation of COVID-19 cases. The study has calculated an infection identification accuracy of $84.10 \%$.
\end{abstract}

Keywords: COVID-19 Identification, Classification, Analysis, Coronavirus, ANN, Convolution Neural Network (CNN).

\section{Introduction}

Coronavirus pandemic has become a serious threat to the whole world since 2019. It was initially seen in the Wuhan region of China. Eventually, it has reached out to 213 countries and infecting millions of people all over the world until today. At present day, it is continuing to infect and take human lives. Hence it became a very important task to detect the virus as quickly as possible before it gets out of control. Initially, the virus showed the symptoms of bitterness in the throat, persistent coughing, fever, and fatigue. These symptoms showed similarities with common flu. Hence it was difficult to identify the coronavirus [1].

It was seen during the X-ray analysis of COVID-19 patients that many differences were observed with the noninfected patient $\mathrm{x}$-rays. Hence, the $\mathrm{x}$-ray images of the patients gave the doctors very important clues on identification and analysis to determine the patients are infected or not [2].

The infection is very viral and travels between individuals by touching and breathing at proximity. It is important to use face masks and keep a safe distance from others in public for preventing the virus from spreading. Treatment of coronavirus needs an intensive amount of care by isolating the patients in hospitals and apply necessary treatments.

Machine learning has become a useful tool to analyze and detect coronavirus patients. It has become more efficient with the help of computer vision and using different machine learning algorithms. Classification is the process of classifying a dataset of different feature extracted data subsets into their class groups. The classification predictive model uses different machine learning algorithms. The most popular algorithms are KNN [3], Naive Bayes [4], Logistic Regression [5], and random forest [6]. 
In this study, a random forest algorithm was used for the feature classification. The random forest algorithm is based on the Classification and Regression Tree (CART) algorithm [7]. Random Forest uses the decision tree of the intuitive model as a series of YES and NO and based on that, it classifies all the data group into its corresponding classes. A dataset of 5000+ x-ray lung images of normal and COVID infected patients is deployed in this study. The classification is carried out by using image processing approaches consist of image filtering ac, image enhancement, and image detection.

\section{Literature survey}

Different studies analyzed the COVID infection data and its spatial and temporal spread. Predictions of the infection growth and the possibility of the cure are developed in biology and medical areas. Machine learning and its algorithms had great assistants to set up the theory of the infection [8]. Machine learning is used as a landmark in automating the task of doctors since the lives of doctors are in danger when it comes to treating the patients. In machine learning, image pre-processing, feature extraction, and classification of the chest x-ray dataset are deployed [9].

Ardabili et al. (2020), presented a comparative analysis of machine learning and soft computing models to predict the COVID-19 with respect to SIR and SEIR. Two machine learning models multi-layer perceptron, (MLP), and adaptive network-based fuzzy inference system, (ANFIS). The paper provided an initial benchmarking to show the potentials of machine learning for future research [10].

Barstugan et al. (2020), gave an insight on early phase detection of COVID-19. The detection was implemented on abdominal CT images. Radiologists detected from CT images that the COVID-19 virus shows different behavior from other viral pneumonia. For detection 4 different data sets are employed. The feature extraction process was applied to increase the classification performance. Grey level matrix and discrete wavelet transform algorithms were used as feature extraction methods. The best classification accuracy was obtained at $99.68 \%$ [11].

Basu et al (2020), proposed a new concept called domain extension transfer learning. In this scenario, machine learning (ML) and Deep Learning (DL) used to have fast and automated detection of abnormalities. Key features of the lung anomalies related to specific signatures of COV $\ddot{A}^{\circ} \mathrm{D}-19$. With pre-trained deep CNN on a large chest, X-ray dataset four classes are classified. The overall accuracy was measured as 95\%, [12].

Zheng et al. (2020), developed a deep learning-based model for automatic COVID-19 detection on chest CTs. A deep learning software system was developed by using 3D CT volumes. The segmented 3D lung region was fed into 3D deep NN to predict the probability of COVID-19 infection. The probability threshold of 0.5 was used to classify COVID (+) or (-). Weakly supervised deep learning models accurately predict the COVID-19 infection probability without annotating the lesions for training [13].

Hemdan et al. (2020) demonstrated the useful application of deep learning models to classify COVID-19 in X-ray images. They proposed the COVIDX-net framework. This includes seven different deep CNN models. Each model has analyzed intensities of the X-ray image to classify patient status either (+) or (-) COVID-19 case, [14].

Wang et al. (2020), introduced COVID-net, a deep CNN design to detect COVID-19 cases from chest X-ray images. They also generated COVIDx, an open-access benchmark dataset from over 10000 patient cases to accelerate the development of accurate deep learning solutions. COVID-net system also carried out predictions based on relevant information from X-ray images [15].

Ozturk et al. (2020), proposed a model using artificial intelligence (AI) techniques coupled with radiological imaging to detect the COVID-19 cases automatically. The model provided accurate diagnostics for binary classification (accuracy of 
98\%) and multi-class classification (accuracy of 87\%). They introduced 17 convolution layers and introduced different filtering on each layer. The model is employed in the initial screening of patients to validate the COVID-19 infection [16] Asif et al. (2020), aimed to detect automatically COVID-19 patients using digital chest X-ray images by using deep CNN. The data set consists of normal, COVID-19, and viral pneumonia chest X-ray images. Inception V3 with Transfer learning is used for the detection of coronavirus pneumonia infected patients using chest X-ray radiographs. Classification accuracy of $98 \%$ was obtained, [17].

In this study, we have also deployed deep learning classification algorithms to predict whether it is a COVID-19 case or not. The algorithm is applied between normal and infected patients. Neural network training is carried out between them and this training is used to detect the new patients.

\section{Material and model}

\subsection{Statistical analysis of dataset}

There are three types of datasets for this statistical analysis. These are training, testing, and validating datasets. Each dataset consists of two types of images: normal non-infected and COVID-19 infected chest $\mathrm{x}$-ray images. The total number of images in the dataset is 5886. The numbers of both normal and infected patients are tabulated in Table 1.

Table 1: Different subset of x-ray dataset

\begin{tabular}{|c|c|c|}
\hline \multirow{2}{*}{ Training dataset } & Normal x-ray data & 1300 images $(48 * 48)$ \\
\cline { 2 - 3 } & Covid infected X-ray & 4900 images $(48 * 48)$ \\
\hline \multirow{2}{*}{ Testing dataset } & Normal x-ray data & 245 images $(48 * 48)$ \\
\cline { 2 - 3 } & Covid infected data & 370 images $(48 * 48)$ \\
\hline \multirow{2}{*}{ Validation dataset } & Normal x-ray data & 20 images $(48 * 48)$ \\
\cline { 2 - 3 } & Covid infected data & 20 images $(48 * 48)$ \\
\hline
\end{tabular}

\subsection{Visual analysis of the data}

Chest X-ray image data classification of the normal non-infected patient and the infected patient was carried out during the selection of images at the start. This gives a great advantage to make the classifier to fit into different machine learning models. The normal image is presented on the left side and the COVID infected image is presented on the right side in Fig.1.

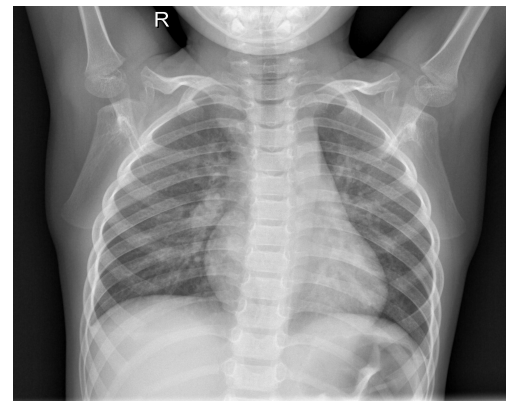

(a)

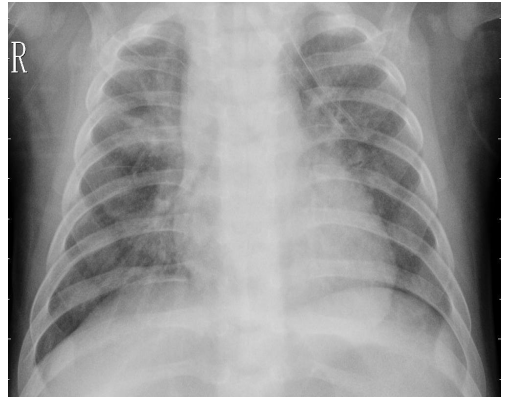

(b)

Fig. 1: (a)Non-infected and (b)infected chest X-ray images. 
Image data for both normal and infected patients are labeled according to Gray levels in the images.[18] It can be seen that the Gray levels of the lower parts of both chest images in Fig.1 seem to have different intensities. The convolution neural network, (CNN), technique [19] is the most used artificial neural network, (ANN), technique in image classification. because of its handling decisions using pooling and convolution layers.

\subsection{Artificial neural network (ANN)}

The ANN is a numerical modeling technique that mimics biological brains. ANN can initially be trained on a sample of the known dataset. It determines the future similar patterns for unknown data which it has never seen before. The ANN can change its structure depending on internal and external information during its flow through the network in the training phase. The ANN can be considered as non-statistical data modeling techniques [20]. They are employed to show the relationship between input and output data.

The ANN is a straightforward network that relates the input and output functions. In this technique, the signals travel through the network between input and output layers without any feedback. The performance of the NN is related to its input-output transfer function. Weights of the neurons reduce the errors between estimated and actual outputs. The sigmoid function is employed for activation during the training stage to transfer the input data to the output layer.

The ANN consist of neurons that are constructed in layers. It generally has one input layer, one output layer. Neurons in the input layers are defined as input data and neurons in the output layer are defined as output data. Hidden layers, between the input and output layers, transform the input data to generate the target output data. The NN calculates the error derivative of the weights and back-propagation algorithm, [21], which is the best method to derive these error derivatives. A typical ANN is seen in Fig. 2.

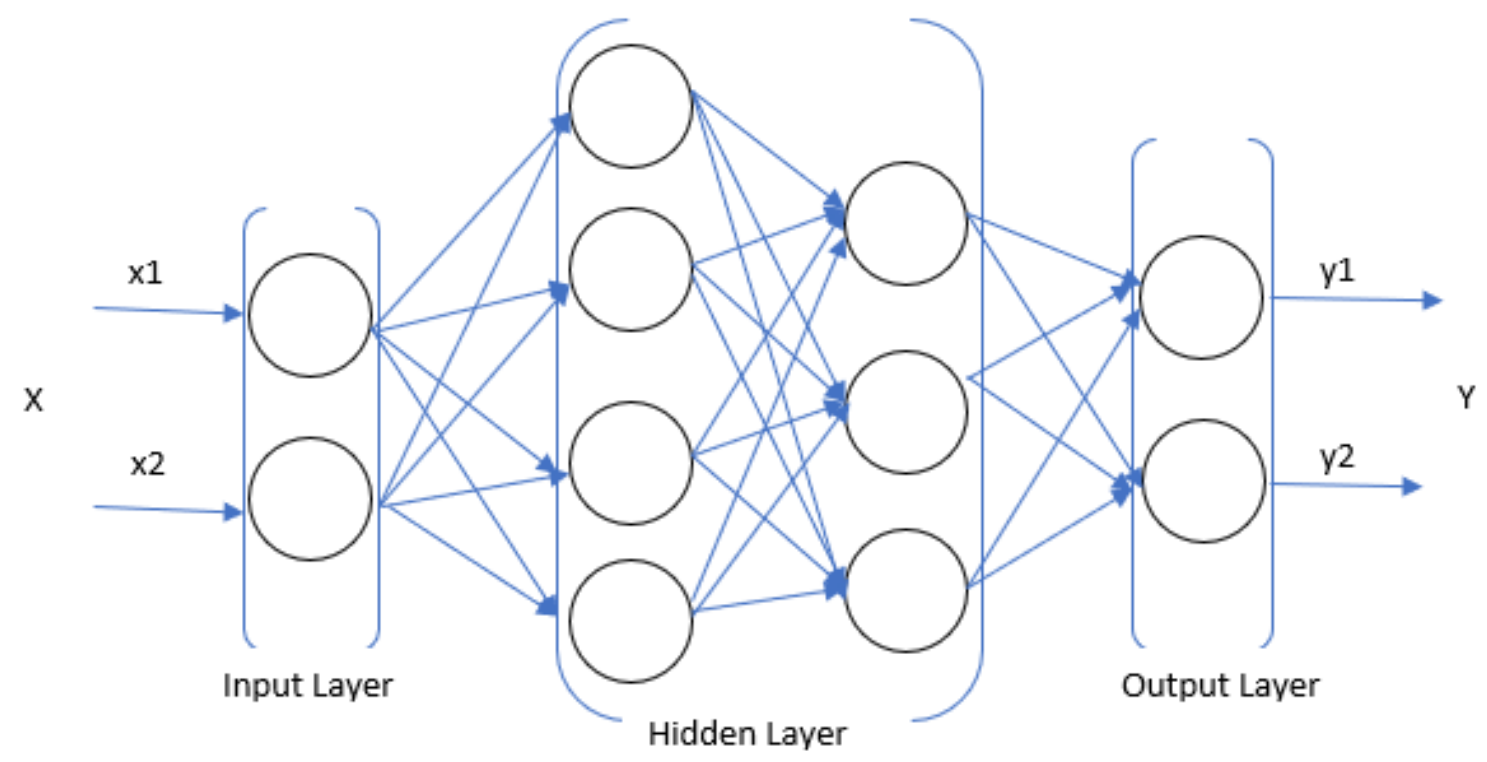

Fig. 2: Basic artificial neural network.

A neural system can be deployed such as a Deep Neural Network (DNN), which utilizes more than one concealed layer. The contrast between the neural system and deep neural system is on the depth or the quantity of concealed layers used in 
the system. Deep Neural Network can be a feed-forward neural system that has more than one hidden layer as shown in Fig. 1 [22]. The training image is taken as the input and after processing the necessary output is produced. Fig. 3 shows the general view of CNN architecture with different functional layers. The first layer is the input layer, the second layer is the convolutional layer, intermediate layers are pooling layers (subsampling) and convolutional layers. The last two layers of the network are the fully connected layer and an output layer.

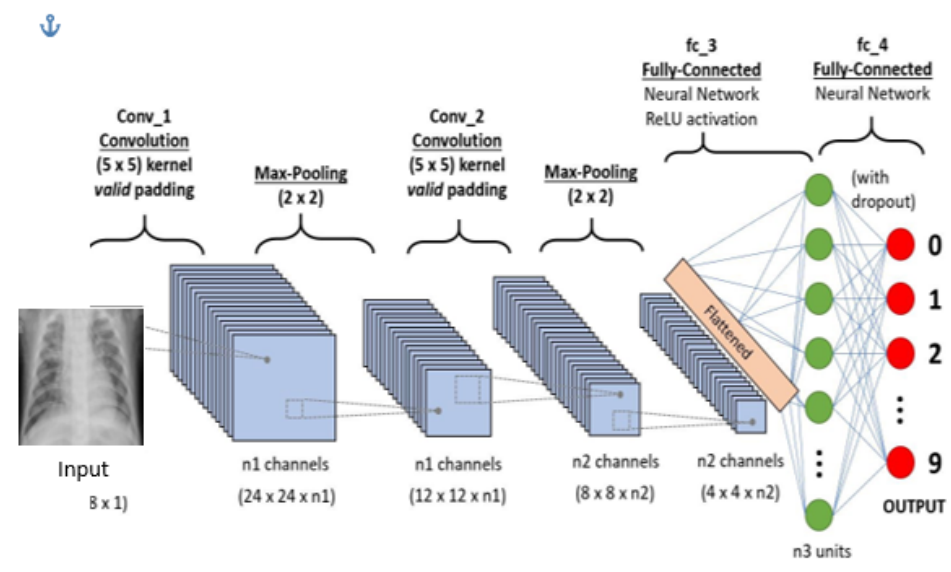

Fig. 3: ANN architecture for image classification.

\subsection{Decision tree}

A decision tree is a tree-like data structure that represents the event in the node and their corresponding decision at the leaf. A decision tree model can be generated with the help of a dataset of training data and a set of validation data to validate the efficiency of the decision tree model. The decision tree is the most mainstream strategy for information mining and effectively comprehending its presentation. The user can unmistakably observe the nodes and branches which display their ingenuity in each decision tree.

The decision tree can be applied to any information types like nominal, ordinal, numerical, etc. It is punctuated inside parentheses. Additionally, Test information orders quick with the assistance of decision tree calculation because of its effortlessness.

\subsection{Adaptive boosting}

Another machine learning algorithm is called AdaBoost. It is developed for the solution of problems faced while boosting a weak classifier into strong classifiers. It can be done by weighting their respective outputs.

The input to the boosting algorithm is the training data in the form of $\mathrm{t}\left(x_{i}, y_{i}\right),\left(x_{m}, y_{m}\right)$ where $x_{i}$ represents instant space $x_{i}$ and $y i$ represents class label set Y. Lets say $Y=0,1$. The AdaBoost algorithm recursively iterates the basic learning technique with t iterations denoted as 1 to $t,[23]$.

Distributing weight set over the entire training dataset is the main objective of the boosting algorithm. In the initial phase, if Dt is the distributed weight of training dataset I on t iterations, all weights are equally distributed. In every iteration, it gradually increases the weight of incorrect classified set and the weak learner is focused more in training the dataset. The pseudocode Algorithm for the integration of CNN and AdaBoost is shown in Table 2. 
Table 2: The pseudocode algorithm for the integration of CNN and AdaBoost

\section{Pseudo code for CNN-AdaBoost}

1. Initialization of data sample with number of training sample.

$\mathrm{D}(i)=1 / n\{i=1,2,3, \ldots n\}$ where $n=$ numberoftrainingsample, $\mathrm{m}$

$=$ totol number of CNN's and D is sample weight

2. Iterate: $m>1$ to $m:$

If $(m==1)$ :

Train the dataset with sample weight $\mathrm{d}_{\mathrm{i}}$

Train_output $=\mathrm{C}^{\wedge} \mathrm{m}-1(\mathrm{k})$ where, $\mathrm{C}$ is $\mathrm{CNN}$ model

Else:

$$
\mathrm{C}^{\wedge} \mathrm{m}(\mathrm{x})=\mathrm{C}^{\wedge} \mathrm{m}-1(\mathrm{x})
$$

3. For $\mathrm{m}^{\text {th }} \mathrm{CNN}$ find class $\left(K_{i}=1,2,3, \ldots . \mathrm{n}\right)$

New_Train_output $=\mathrm{C}^{\wedge} \mathrm{m}(\mathrm{x})$ for $\mathrm{D}(\mathrm{i})$

4. $D_{m}=P_{k}^{(m)}(x)$

$P_{k}^{(m)}(x)$ where $P$ is the estimated Class for $X$ data

5. Re- Normalization of data weight sample.

6. Save $\mathrm{C}^{\mathrm{m}}(\mathrm{x}) \mathrm{CNN}$.

\section{Experimental methodology}

The main goal of this study is to present the highest quality classifier using deep learning. In this methodology, several steps are included as shown in Fig. 4. It consists of image filtration and image filtering, pooling, and training for multiple epochs.

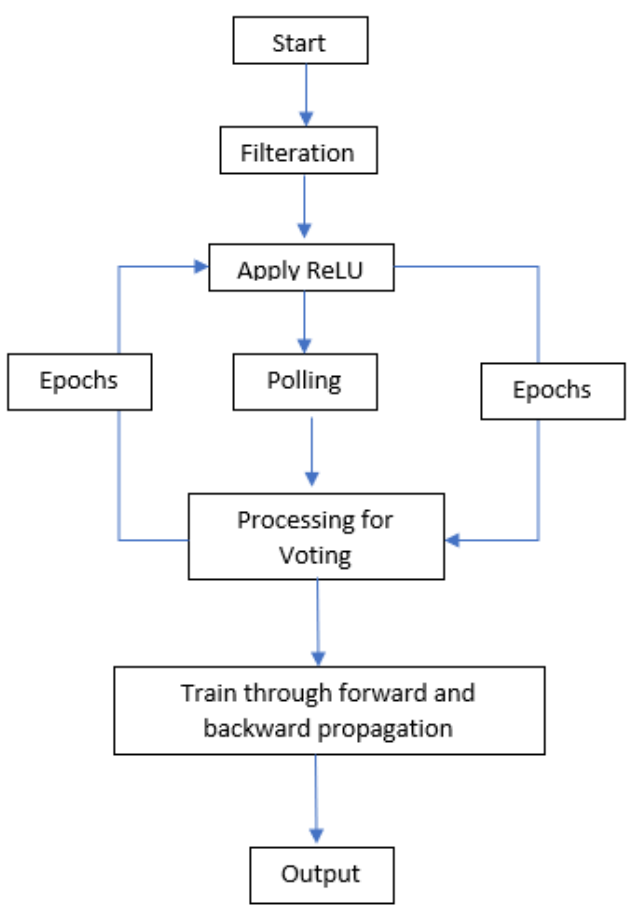

Fig. 4: Methodology. 


\subsection{Image filtration}

Image filtration plays a significant role when it comes to classifying the images. Image filtration or feature map is carried out with the help of ReLU and SoftMax activation function [24]. The feature map or activation map will help to detect features in input images like horizontal and vertical lines, curves, edge, etc. Different filter maps are shown in Fig 5.In a deep learning approach, there are several kinds of activation functions [25]. The list and comparison between different activation functions are shown in Fig.6 and Fig.7.
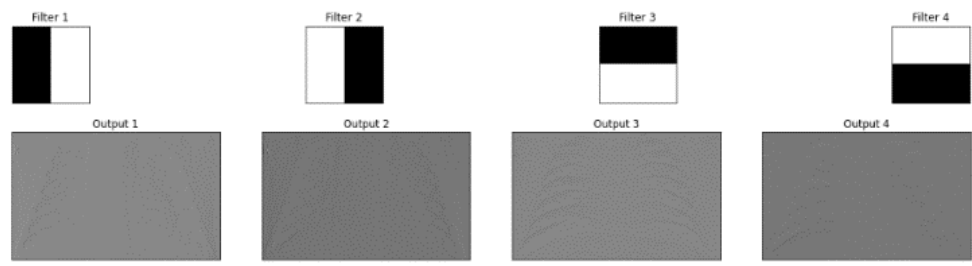

Fig. 5: Activation map or filter map.

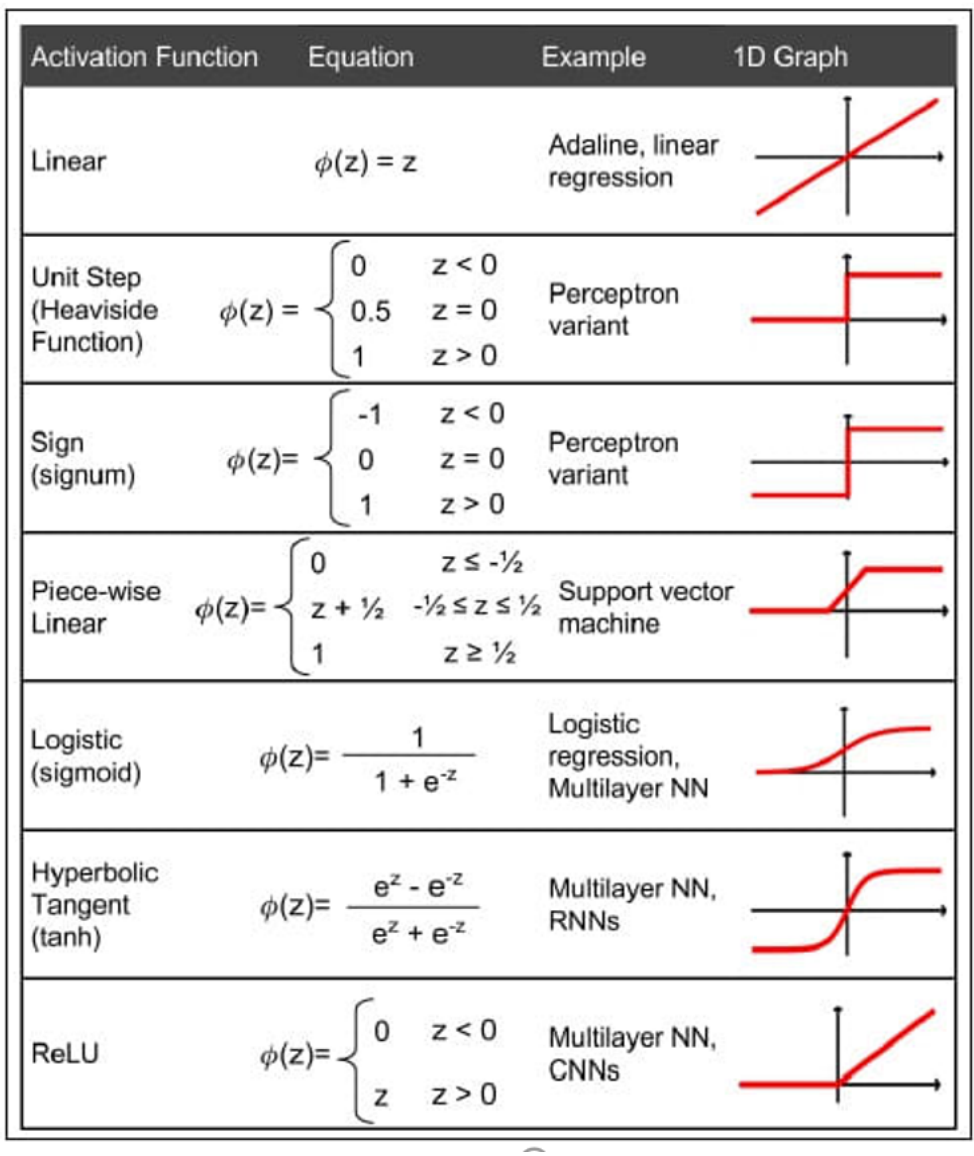

Fig. 6: Different Activation functions. 


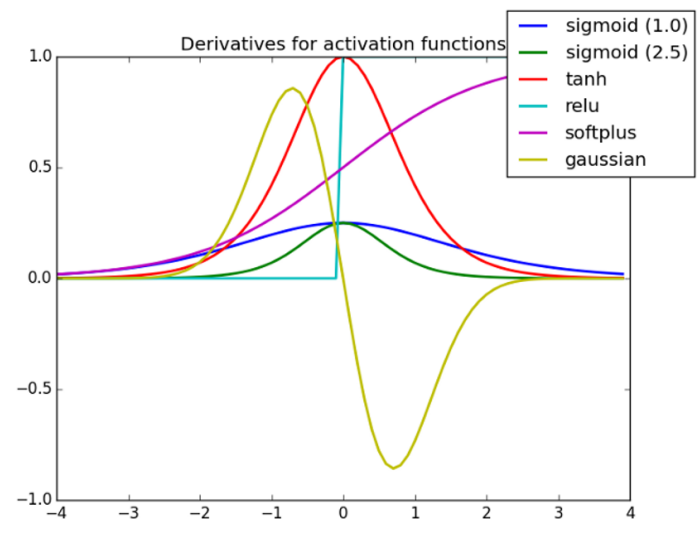

Fig. 7: Difference between multiple activation functions.

\subsection{Pooling layer}

After the feature mapping and filter mapping, the processed images arrive at the pooling layer [26]. Pooling is done for invariance to translation. Pooling is applied when a slight change is intended over the input image without changing the output. Pooling factors can be calculated in several ways such as minimum pooling, average pooling, and maximum pooling. These pooling factors will help to provide comparatively better performances.

\subsection{Deep convolutional neural network model}

Creating a deep neural network model is a major role in developing a classifier. An accurate and efficient model can be built by having multiple configurations of parameters in the model. Applying the activation function plays a vital role in making an efficient deep learning model. In this model, 4 convolution layers are used with the parameters of (8,3,padding='same', activation='relu',),(16,3,padding='same', activation='relu',),(64,3,padding='same',

activation='relu',),(512,3,padding='same', activation='relu',) using the dropout parameter of 0.3 as shown in Fig.8. The configuration of CNN model plays a vital role in developing and integrating AdaBoost algorithm. See Table 3.

Table 3: Configuration of CNN.

\begin{tabular}{|c|c|}
\hline Layers & Configurations \\
\hline 2D Convolution & 32 filters, 3x1 kernal and ReLU \\
\hline Max-Pooling & $4 \times 1$ kernal \\
\hline Dropout & $0.2(\% 20)$ \\
\hline Fully Connected & 256 Neurons, ReLU \\
\hline Dropout & $0.2(\% 20)$ \\
\hline Fully Connected & 128 Neurons, ReLU \\
\hline Fully Connected & 64 Neurons, SoftMax \\
\hline Dropout & $0.2(\% 20)$ \\
\hline Fully Connected & 32 Neurons, SoftMax \\
\hline
\end{tabular}

\subsection{Visualization of feature maps or filter maps}

Feature map or filter maps are generated with the help of a previously used convolution neural network layer. The visualization of an input image through a convolution network is essential to give an insight view over what is 
Model: "sequential_3"

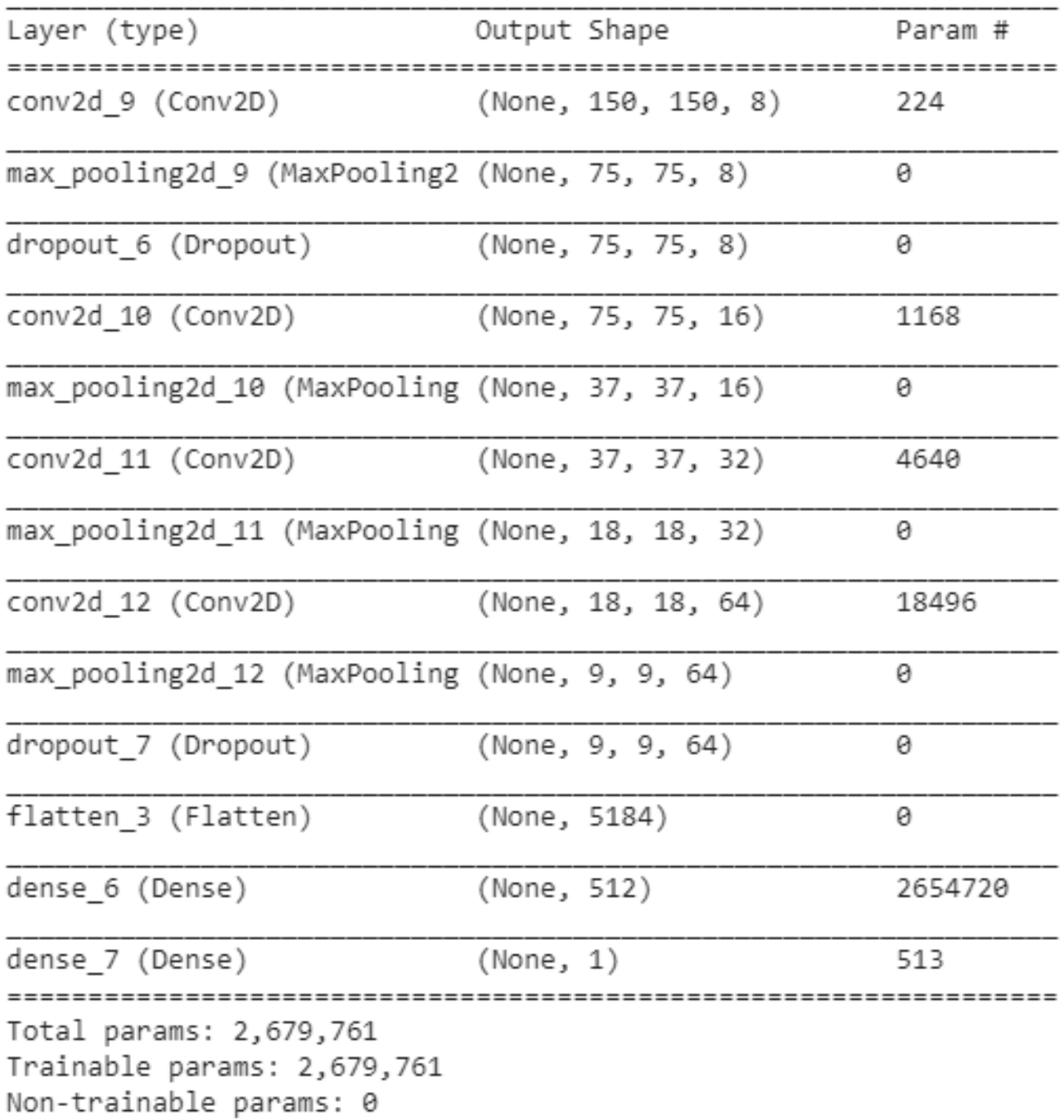

Fig. 8: Neural network model summary.

happening. It will help to know the internal representation of convolution network layer architecture.

Algorithm to generate a visualization of filter maps is tabulated below:

(1) Make the model by taking image as input, output, and its feature map.

(2) Load input Image.

(3) With the help of NumPy library, convert input image into NumPy array.

(4) Normalize the input image array.

(5) Train and run the model with the input image array.

(6) With the help of matplotlib library, make a plot for all convolution layers.

The visualization of filter maps is shown in Fig.9. 


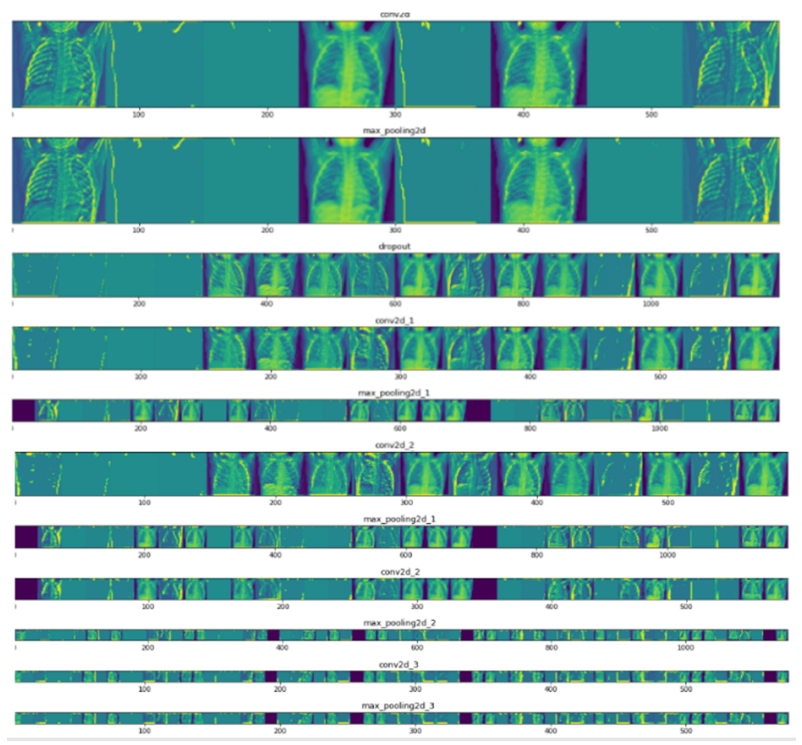

Fig. 9: Visualization of filter map.

\section{Conclusion and results}

COVID-19 was initially experienced in the Wuhan area of China and has been compromising the general well being and the economy of the world. The infection shows somewhat comparable practices with other viral pneumonia. Subsequently, the spreading speed of the infection made it difficult to find a quick vaccine. X-ray imaging of the COVID-19 patients shows various new symptoms as indicated during clinical examinations. Some of these symptoms were bronchiectasis, sore expanding side effects, and diverse shadowiness in X-ray images. give to analyzed COVID-19, without any problem. Training dataset and their corresponding percentages are calculated by detecting their corresponding classes.in the proposed methodology in Table 4.

Table 4: Sample training dataset.

\begin{tabular}{|c|c|c|}
\hline \#Sample & Percentage & Class \\
\hline Training & & \\
\hline 1600 & $46.74 \%$ & 0 (not infected) \\
\hline 2159 & $53.26 \%$ & 1 (infected) \\
\hline Testing & & \\
\hline 1126 & $42.36 \%$ & 0 (not infected) \\
\hline 2174 & $57.64 \%$ & 1 (infected) \\
\hline
\end{tabular}

In this study, the coronavirus image set has diverse sorts of images, which were gained with various X-ray devices. In this way, five-element extraction techniques were used to discover the list of capabilities that isolates the tainted patches with high precision. The dataset in this examination was framed physically and accomplished $99.76 \%$ characterization accuracy. The proposed strategy can be tried on another coronavirus X-ray image dataset soon. Detected filtered image is shown in Fig. 10. 


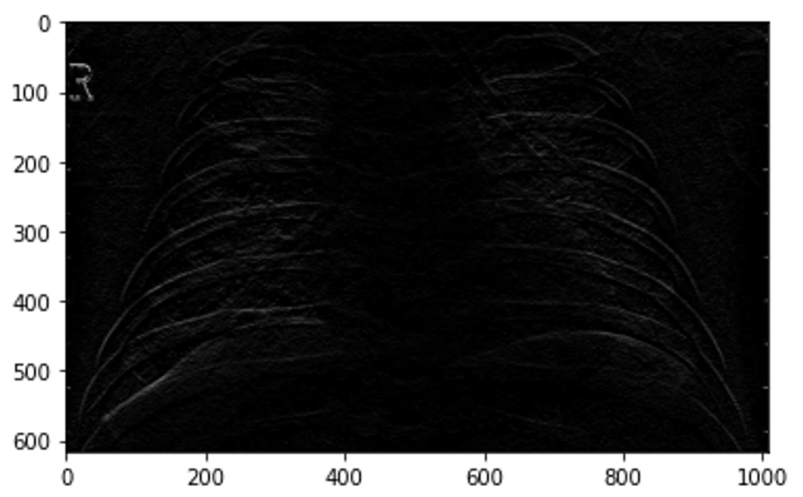

Fig. 10: Detected infected $x$-ray image.

The study examines most of the clinical examinations. The characterization accuracy was found high similar to leading studies explained in the Literature survey. This investigation inspected COVID-19 infected images for characterization purposes. There ought to be more categorization order and division in characterization on the accurate detection studies. The accuracy of proposed AdaBoost integrated with CNN gives better output than CNN as in Table 5.

Table 5: Proposed AdaBoost CNN accuracy for COVID-19 dataset.

\begin{tabular}{|c|c|c|c|}
\hline Training Accuracy (\%) & Testing Accuracy (\%) & \# Estimator (m) & \#epochs \\
\hline 99.76 & 84.10 & 2 & 25 \\
\hline 97.42 & 79.26 & 2 & 25 \\
\hline 86.22 & 71.19 & 3 & 10 \\
\hline 74.40 & 65.43 & 10 & 5 \\
\hline
\end{tabular}

Additionally, the dataset preparations should be expanded with more details. The AI techniques ought to be executed more on X-ray abdominal images, X-ray chest images, and blood test results. Finally, this study produced a very good characterization accuracy result similar to previously released results.

\section{Competing interests}

The authors declare that they have no competing interests.

\section{Authors' contributions}

All authors have contributed to all parts of the article. All authors read and approved the final manuscript.

\section{References}

[1] O. Arrizabalaga, D. Otaegui, I. Vergara, J. Arrizabalaga, and E. MÃ@ndez, Open Access of COVID-19-related publications in the first quarter of 2020: a preliminary study based in PubMed, F1000Res, vol. 9, p. 649, Jun. 2020.

[2] R. M. Pereira, D. Bertolini, L. O. Teixeira, C. N. Silla, and Y. M. G. Costa, COVID-19 identification in chest X-ray images on flat and hierarchical classification scenarios, Computer Methods and Programs in Biomedicine, vol. 194, p. 105532, Oct. 2020.

[3] W. M. Shaban, A. H. Rabie, A. I. Saleh, and M. A. Abo-Elsoud, A new COVID-19 Patients Detection Strategy (CPDS) based on hybrid feature selection and enhanced KNN classifier, Knowledge-Based Systems, vol. 205, p. 106270, Oct. 2020. 
[4] J. Chen, H. Huang, S. Tian, and Y. Qu, Feature selection for text classification with $\mathrm{Na}^{-}{ }^{-}$ve Bayes, Expert Systems with Applications, vol. 36, no. 3, pp. 5432-5435, Apr. 2009.

[5] K. Xu et al., Application of ordinal logistic regression analysis to identify the determinants of illness severity of COVID-19 in China, Epidemiol. Infect., vol. 148, p. e146, 2020.

[6] C. Iwendi et al., COVID-19 Patient Health Prediction Using Boosted Random Forest Algorithm, Front. Public Health, vol. 8, p. 357, Jul. 2020.

[7] R. K. Zimmerman et al., Classification and Regression Tree (CART) analysis to predict influenza in primary care patients, BMC Infect Dis, vol. 16, no. 1, p. 503, Dec. 2016.

[8] R. Sujatha, J. M. Chatterjee, and A. E. Hassanien, Correction to: A machine learning forecasting model for COVID-19 pandemic in India, Stoch Environ Res Risk Assess, vol. 34, no. 10, pp. 1681-1681, Oct. 2020.

[9] F. Yang et al., Feature Extraction and Classification on Esophageal X-Ray Images of Xinjiang Kazak Nationality, Journal of Healthcare Engineering, vol. 2017, pp. 1-11, 2017.

[10] S. F. Ardabili et al., COVID-19 Outbreak Prediction with Machine Learning, Health Informatics, preprint, Apr. 2020.

[11] M. Barstugan, U. Ozkaya, and S. Ozturk, Coronavirus (COVID-19) Classification using CT Images by Machine Learning Methods, arXiv:2003.09424 [cs, eess, stat], Mar. 2020, Accessed: Mar. 24, 2021.

[12] S. Basu, S. Mitra, and N. Saha, Deep Learning for Screening COVID-19 using Chest X-Ray Images, Radiology and Imaging, preprint, May 2020.

[13] C. Zheng et al., Deep Learning-based Detection for COVID-19 from Chest CT using Weak Label, Infectious Diseases (except HIV/AIDS), preprint, Mar. 2020.

[14] E. E.-D. Hemdan, M. A. Shouman, and M. E. Karar, COVIDX-Net: A Framework of Deep Learning Classifiers to Diagnose COVID-19 in X-Ray Images, arXiv:2003.11055 [cs, eess], Mar. 2020.

[15] L. Wang and A. Wong, COVID-Net: A Tailored Deep Convolutional Neural Network Design for Detection of COVID-19 Cases from Chest X-Ray Images, arXiv:2003.09871 [cs, eess], May 2020.

[16] T. Ozturk, M. Talo, E. A. Yildirim, U. B. Baloglu, O. Yildirim, and U. Rajendra Acharya, Automated detection of COVID-19 cases using deep neural networks with X-ray images, Computers in Biology and Medicine, vol. 121, p. 103792, Jun. 2020.

[17] S. Asif, Y. Wenhui, H. Jin, Y. Tao, and S. Jinhai, Classification of COVID-19 from Chest X-ray images using Deep Convolutional Neural Networks, Radiology and Imaging, preprint, May 2020.

[18] M. E. H. Chowdhury et al., Can AI Help in Screening Viral and COVID-19 Pneumonia?, IEEE Access, vol. 8, pp. 132665-132676, 2020

[19] D. Singh, V. Kumar, Vaishali, and M. Kaur, Classification of COVID-19 patients from chest CT images using multi-objective differential evolution?based convolutional neural networks, Eur J Clin Microbiol Infect Dis, vol. 39, no. 7, pp. 1379-1389, Jul. 2020

[20] D. J. Sargent, Comparison of Artificial Neural Networks with OtherStatistical Approaches, presented at the Conference on Prognostic Factors and Staging in CancerManagement: Contributions of Artificial Neural Networksand Other Statistical Methods.

[21] J. A. Cruz-Lopez, V. Boyer, and D. El-Baz, Training Many Neural Networks in Parallel via Back-Propagation, in 2017 IEEE International Parallel and Distributed Processing Symposium Workshops (IPDPSW), Orlando / Buena Vista, FL, USA, May 2017, pp. 501-509.

[22] A. B. Adege et al., Applying Deep Neural Network (DNN) for large-scale indoor localization using feed-forward neural network (FFNN) algorithm, in 2018 IEEE International Conference on Applied System Invention (ICASI), Chiba, Apr. 2018, pp. 814-817,

[23] K. W. Walker and Z. Jiang, Application of adaptive boosting (AdaBoost) in demand-driven acquisition (DDA) prediction: A machine-learning approach, The Journal of Academic Librarianship, vol. 45, no. 3, pp. 203-212, May 2019.

[24] T. Mahmud, M. A. Rahman, and S. A. Fattah, CovXNet: A multi-dilation convolutional neural network for automatic COVID19 and other pneumonia detection from chest X-ray images with transferable multi-receptive feature optimization, Computers in Biology and Medicine, vol. 122, p. 103869, Jul. 2020.

[25] Y. Wang, Y. Li, Y. Song, and X. Rong, The Influence of the Activation Function in a Convolution Neural Network Model of Facial Expression Recognition, Applied Sciences, vol. 10, no. 5, p. 1897, Mar. 2020.

[26] A. Kumar, Ordinal Pooling Networks: For Preserving Information over Shrinking Feature Maps, arXiv:1804.02702 [cs], Apr. 2018. 